\title{
Free Transverse Vibration of Orthotropic Thin Trapezoidal Plate of Parabolically Varying Thickness Subjected to Linear Temperature Distribution
}

\author{
Arun Kumar Gupta and Shanu Sharma \\ Department of Mathematics, M.S. College, Saharanpur 247001, India \\ Correspondence should be addressed to Arun Kumar Gupta; gupta_arunnitin@yahoo.co.in
}

Received 21 October 2013; Revised 4 March 2014; Accepted 20 March 2014; Published 3 April 2014

Academic Editor: Anindya Ghoshal

Copyright (C) 2014 A. K. Gupta and S. Sharma. This is an open access article distributed under the Creative Commons Attribution License, which permits unrestricted use, distribution, and reproduction in any medium, provided the original work is properly cited.

\begin{abstract}
The present paper deals with the free transverse vibration of orthotropic thin trapezoidal plate of parabolically varying thickness in $x$-direction subjected to linear temperature distribution in $x$-direction through a numerical method. The deflection function is defined by the product of the equations of the prescribed continuous piecewise boundary shape. Rayleigh-Ritz method is used to evaluate the fundamental frequencies. The equations of motion, governing the free transverse vibrations of orthotropic thin trapezoidal plates, are derived with boundary condition CSCS. Frequency corresponding to the first two modes of vibration is calculated for the orthotropic thin trapezoidal plate having CSCS edges for different values of thermal gradient, taper constant, and aspect ratio. The proposed method is applied to solve orthotropic thin trapezoidal plate of variable thickness with C-S-C-S boundary conditions. Results are shown by figures for different values of thermal gradient, taper constant, and aspect ratio for the first two modes of vibrations.
\end{abstract}

\section{Introduction}

Plate theory has been applied to reduce vibration and noise in structures since the end of the 19th century where it began with the work of German physicist Chladni, who discovered various modes of free vibrations experimentally. Since then, it has developed into an escalating and expansive field with a wide variety of theoretical and empirical techniques, dealing with increasingly complicated problems. In marine and aerospace engineering fields, where lightweight structural elements with orthotropic materials are of primary importance, orthotropic trapezoidal plate has extensive application. They are essentially thin plate structures which provide enhanced stiffness and stability characteristics. They also have widespread application in many other branches of modern civil, mechanical, and structural engineering and, in these applications, they are regularly subjected to static and time varying loads. Hence, analysis of orthotropic trapezoidal plate under different conditions has always been an area of immense interest to researchers. Research work on the dynamic characteristics of orthotropic trapezoidal plate has a long history as different researchers have employed different methodologies to perform dynamic analysis of such structures.

Plates of variable thickness are used in nuclear reactor structures, naval structures, and aeronautical fields, electromechanical transducers for the electronic telephones, and mirrors and lenses in optical system. The practical importance of these plates has made vibration analysis essential for avoiding resonance excited by internal or external forces. Also, in recent years, the development of solid propellant rocket motors and the increased use of soft filaments in aerospace structure and high speed runways, many practical solid structure interaction problems such as floor slabs of multistory buildings and buildings activities in cold regions have intensified the need for solution of various problems of plates and other structures supported on elastic media.

The literature on the free vibrations of plates is vast. A number of researchers have worked on free vibration analysis of rectangular, circular, elliptical, and so forth, plates and 


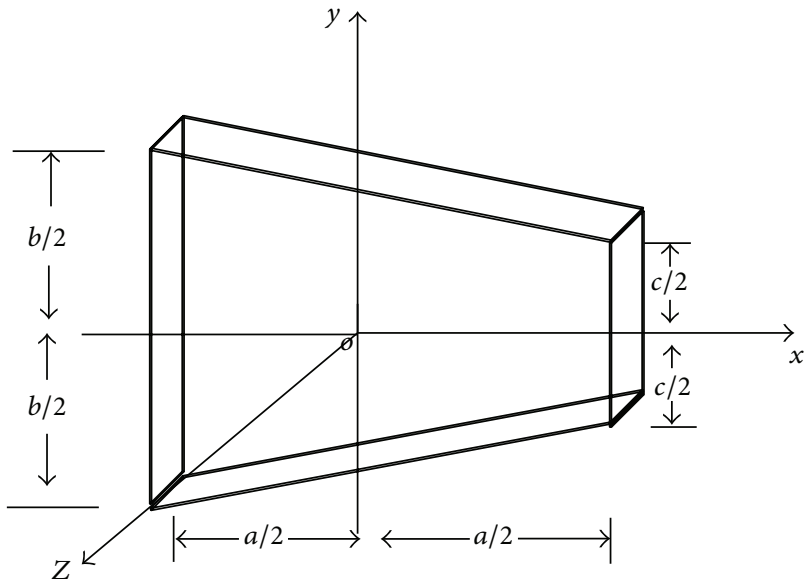

(a)

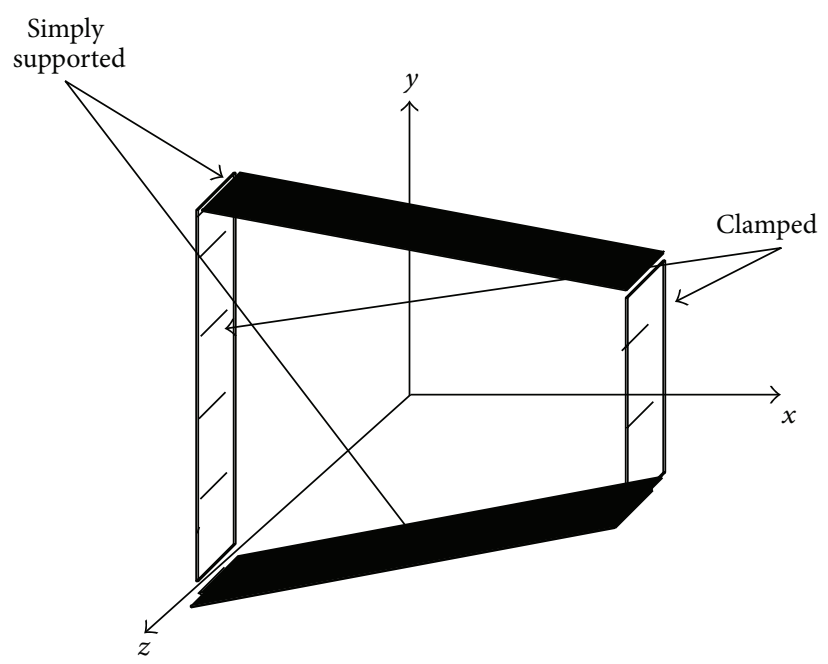

(b)

FIGURE 1: (a) The geometry of symmetry trapezoidal thin plate; (b) boundary conditions for the plate.

variable thickness. Little research work has been done on nonuniform thickness trapezoidal plates as compared to the other plates.

Zenkour [1] studied an exact solution for bending thin rectangular plates with uniform, linear, and quadratic thickness variations. Tomar et al. [2] attempted a problem of axisymmetric vibrations of an isotropic elastic nonhomogeneous circular plate of linearly varying thickness. Tomar et al. [3] studied features of the free vibration of an isotropic nonhomogeneous infinite plate of parabolically varying thickness. Srinivas et al. [4] did an exact analysis for vibration of simply supported homogeneous and laminated thick rectangular plates. Saliba [5] considered the transverse free vibration of fully clamped symmetrical trapezoidal plates. Orris and Petyt [6] discussed the finite element method of the vibration of trapezoidal plates. Qatu [7] studied the natural frequencies for cantilevered laminated composite right triangular and trapezoidal plates. Kitipornchai et al. [8] discussed the problem of vibrations of thick trapezoidal plates. Recently, Gupta and Sharma [9] studied the thermal effect on free vibration of orthotropic thin trapezoidal plate of linearly varying thickness.

This research paper proposes a new analytical model for the vibration analysis of an aircraft panel structure modeled as a homogeneous orthotropic thin trapezoidal plate and based on classical plate theory with boundary conditions including CSCS one, which is the extension of author's previous work [9]. Plate is assumed to be thin, orthotropic, and homogeneous in nature. Thickness of the plate is varying parabolically in $x$-direction. Modulus of elasticity of the plate material varies linearly with temperature. Under the influence of linearly transient distributed temperature field, it is assumed that Young's modulus and shear modulus of plate material varied linearly along $x$-axis. The governing differential equation of motion of the orthotropic trapezoidal plate is solved by using Rayleigh-Ritz method.

The frequencies for the first and second mode of vibration are calculated for the thin trapezoidal plate having CSCS edges for the different values of taper constant, thermal gradient, and aspect ratio presented in graphical form.

\section{Method of Analysis}

Consider a flat, thin, and homogenous orthotropic trapezoidal plate as shown in Figure 1(a). The orthotropic plate with symmetric parabolically varying thickness and symmetric trapezoidal plan form is taken. The thickness $h_{0}$ is along the edge $x=-a / 2$ and $\beta h_{0}$ is the plate thickness at the edge $x=a / 2$, where $\beta$ is the thickness variation ratio, that is, taper constant.

The thickness variation of plate can be expressed as

$$
h(x)=h_{0} g(x),
$$

where $g(x)$ is the thickness variation function.

Introducing nondimensional coordinate system as $\xi=$ $x / a, \eta=y / b$, where $a$ and $b$ are the span and width of the plate plan form as shown in Figure 1(a).

Let us assume that orthotropic trapezoidal plate under consideration is subjected to steady one-dimensional temperature distribution $\tau$ along the length; therefore, one can take $\tau$ as

$$
\tau=\tau_{0}\left(\frac{1}{2}-\xi\right),
$$

where $\tau_{0}$ is the temperature at $\xi=-1 / 2,-1 / 2 \leq \xi \leq 1 / 2$, and $\tau_{0} \leq \tau(\xi) \leq 0$.

For most orthotropic materials, modulus of elasticity is described as a function of temperature as

$$
\begin{gathered}
E_{\xi}(\tau)=E_{1}(1-\gamma \tau), \\
E_{\eta}=E_{2}(1-\gamma \tau), \\
G_{\xi \eta}(\tau)=G_{0}(1-\gamma \tau),
\end{gathered}
$$

where $E_{\xi}$ and $E_{\eta}$ are Young's moduli in $x$-direction and $y$ direction, respectively, and $G_{\xi \eta}$ is the shear modulus, $\gamma$ is slope 
of vibration of moduli with temperature, and $E_{1}, E_{2}$, and $G_{0}$ are the values of moduli at some reference temperature; that is, $\tau=0$.

Using (2), (3) becomes

$$
\begin{aligned}
E_{\xi} & =E_{1}\left[1-\alpha\left(\frac{1}{2}-\xi\right)\right], \\
E_{\eta} & =E_{2}\left[1-\alpha\left(\frac{1}{2}-\xi\right)\right], \\
G_{\xi \eta} & =G_{0}\left[1-\alpha\left(\frac{1}{2}-\xi\right)\right],
\end{aligned}
$$

where $\alpha=\gamma \tau_{0}(0 \leq \alpha<1)$, a parameter known as thermal gradient.

Flexural and torsion rigidity is given by Leissa [10] as

$$
\begin{gathered}
D_{\xi}=\frac{E_{\xi} h^{3}}{12\left(1-\nu_{\eta} \nu_{\xi}\right)}, \\
D_{\eta}=\frac{E_{\eta} h^{3}}{12\left(1-\nu_{\eta} \nu_{\xi}\right)}, \\
D_{\xi \eta}=\frac{G_{\xi \eta} h^{3}}{12} .
\end{gathered}
$$

By using (4) in (5), expression for flexural rigidity and torsion rigidity becomes

$$
\begin{gathered}
D_{\xi}=\frac{E_{1} h^{3}[1-\alpha((1 / 2)-\xi)]}{12\left(1-v_{\xi} v_{\eta}\right)}, \\
D_{\eta}=\frac{E_{2} h^{3}[1-\alpha((1 / 2)-\xi)]}{12\left(1-v_{\xi} \nu_{\eta}\right)}, \\
D_{\xi \mu}=\frac{G_{0} h^{3}[1-\alpha((1 / 2)-\xi)]}{12} .
\end{gathered}
$$

Also

$$
D_{1}=v_{\eta} D_{\xi}=v_{\xi} D_{\eta}
$$

where $h$ is the thickness of the plate.

With the assumption of small free vibration, the displacement function $w$ is periodic in time, so it can be expressed as

$$
w(x, y, t)=W(x, y) \sin \omega t
$$

where $\omega$ denotes the frequency of vibration.

The nondimensional thickness variation function can be expressed as

$$
g(\xi)=\left[1-(1-\beta)\left(\xi+\frac{1}{2}\right)^{2}\right], \quad g(\xi) \geq 0,
$$

where $\beta$ is the taper constant.
The expression for the strain energy $V$ and kinetic energy $T$ in the plate is given by

$$
\begin{array}{r}
V=\frac{a b}{2} \iint\left[D_{\xi}\left(\frac{\partial^{2} W}{\partial \xi^{2}}\right)^{2}+D_{\eta}\left(\frac{\partial^{2} W}{\partial \eta^{2}}\right)^{2}\right. \\
+2 D_{1}\left(\frac{\partial^{2} W}{\partial \xi^{2}}\right)\left(\frac{\partial^{2} W}{\partial \eta^{2}}\right) \\
\left.+4 D_{\xi \eta}\left(\frac{\partial^{2} W}{\partial \xi \partial \eta}\right)^{2}\right] d \xi d \eta \\
T=\frac{a b}{2} \rho \omega^{2} h_{0} \iint g(\xi) W^{2} d \xi d \eta .
\end{array}
$$

Using (1), (6), (7), and (9) in (10), one has

$$
\begin{gathered}
V=\frac{a b}{2} \frac{E_{1} h_{0}^{3}}{12\left(1-\nu_{\xi} \nu_{\eta}\right)} \\
\times \iint\left[1-\alpha\left(\frac{1}{2}-\xi\right)\right]\left[1-(1-\beta)\left(\xi+\frac{1}{2}\right)^{2}\right]^{3} \\
\times\left(\left(\frac{\partial^{2} W}{\partial \xi^{2}}\right)^{2}+\frac{E_{2}}{E_{1}}\left(\frac{\partial^{2} W}{\partial \eta^{2}}\right)^{2}\right. \\
+2 \nu_{\xi}\left(\frac{\partial^{2} W}{\partial \xi^{2}}\right)\left(\frac{\partial^{2} W}{\partial \eta^{2}}\right) \\
\left.+4 \frac{G_{0}\left(1-\nu_{\xi} \nu_{\eta}\right)}{E_{1}}\left(\frac{\partial^{2} W}{\partial \xi \partial \eta}\right)^{2}\right) d \xi d \eta, \\
T=\frac{a b}{2} \rho \omega^{2} h_{0} \iint\left[1-(1-\beta)\left(\xi+\frac{1}{2}\right)^{2}\right] W^{2} d \xi d \eta .
\end{gathered}
$$

The two-term deflection function taken as [11] which satisfies the boundary condition of the plate considered

$$
\begin{aligned}
W=\left[\xi+\frac{1}{2}\right]^{2}\left[\eta-\left(\frac{b-c}{2}\right) \xi+\left(\frac{b+c}{4}\right)\right] \\
\times \\
\times\left[\xi-\frac{1}{2}\right]^{2}\left[\eta+\left(\frac{b-c}{2}\right) \xi-\left(\frac{b+c}{4}\right)\right] \\
\times\left[A_{1}+A_{2}\left(\xi+\frac{1}{2}\right)\left(\eta-\left(\frac{b-c}{2}\right) \xi+\left(\frac{b+c}{4}\right)\right)\right. \\
\left.\times\left(\xi-\frac{1}{2}\right)\left(\eta+\left(\frac{b-c}{2}\right) \xi-\left(\frac{b+c}{4}\right)\right)\right],
\end{aligned}
$$

where $A_{1}$ and $A_{2}$ are constants.

Equation (12) is taken to satisfy boundary condition on trial basis and provides a good estimation to the frequency for first mode if one takes two-term function.

An approximate solution to the problem is given by using the Rayleigh-Ritz method. According to this method, maximum strain energy must be equal to maximum kinetic energy. To apply Rayleigh-Ritz technique, one must has

$$
\delta(F)=0 .
$$


The energy functional for the plate is

$$
F=V-T .
$$

Using (11) in (14), we have

$$
\delta\left(V_{1}-\lambda^{2} T_{1}\right)=0
$$

where

$$
\begin{gathered}
V_{1}=\iint\left[1-\alpha\left(\frac{1}{2}-\xi\right)\right]\left[1-(1-\beta)\left(\xi+\frac{1}{2}\right)^{2}\right]^{3} \\
\times\left(\left(\frac{\partial^{2} W}{\partial \xi^{2}}\right)^{2}+\frac{E_{2}}{E_{1}}\left(\frac{\partial^{2} W}{\partial \eta^{2}}\right)^{2}\right. \\
+2 v_{\xi} \frac{E_{2}}{E_{1}}\left(\frac{\partial^{2} W}{\partial \xi^{2}}\right)\left(\frac{\partial^{2} W}{\partial \eta^{2}}\right) \\
\left.+4 \frac{G_{0}\left(1-\nu_{\xi} \nu_{\eta}\right)}{E_{1}}\left(\frac{\partial^{2} W}{\partial \xi \partial \eta}\right)^{2}\right) d \xi d \eta \\
T_{1}=\iint\left[1-(1-\beta)\left(\xi+\frac{1}{2}\right)^{2}\right] W^{2} d \xi d \eta \\
\lambda^{2}=\frac{12 \rho_{0} \omega^{2} a^{5}\left(1-\nu_{\xi} \nu_{\eta}\right)}{E_{1} h_{0}^{2}}
\end{gathered}
$$

is a frequency parameter.

Two edges of the plates are clamped and two are simply supported; that is, all of the four degrees of freedom of the nodes to the side faces of the plates are constrained as shown in Figure 1(b).

Here limit of $\eta$ is $-(b+c) / 4 b-((c-b) a / 2 b) \xi$ to $(b+c) / 4 b+$ $((c-b) a / 2 b) \xi$, and limit of $\xi$ is -0.5 to 0.5 .

Equation (15) contains two unknown constants $A_{1}$ and $A_{2}$ which can be evaluated as

$$
\frac{\partial\left(V_{1}-\lambda^{2} T_{1}\right)}{\partial A_{n}}=0, \quad n=1,2 .
$$

Equation (17) simplifies to the form

$$
c_{n 1} A_{1}+c_{n 2} A_{2}=0, \quad n=1,2,
$$

where $c_{n 1}$ and $c_{n 2}(n=1,2)$ involve parametric constants and frequency parameter.

For a nonzero solution, the coefficient matrix of (18) must be zero. In this way, the frequency equation must come out to be

$$
\left|\begin{array}{ll}
c_{11} & c_{12} \\
c_{21} & c_{22}
\end{array}\right|=0
$$

From (19), one can obtain a quadratic equation in $\lambda^{2}$ from which two values of $\lambda^{2}$ can be found.

\section{Results and Discussions}

Here numerical results for an orthotropic trapezoidal plate of parabolically varying thickness are calculated. Frequencies

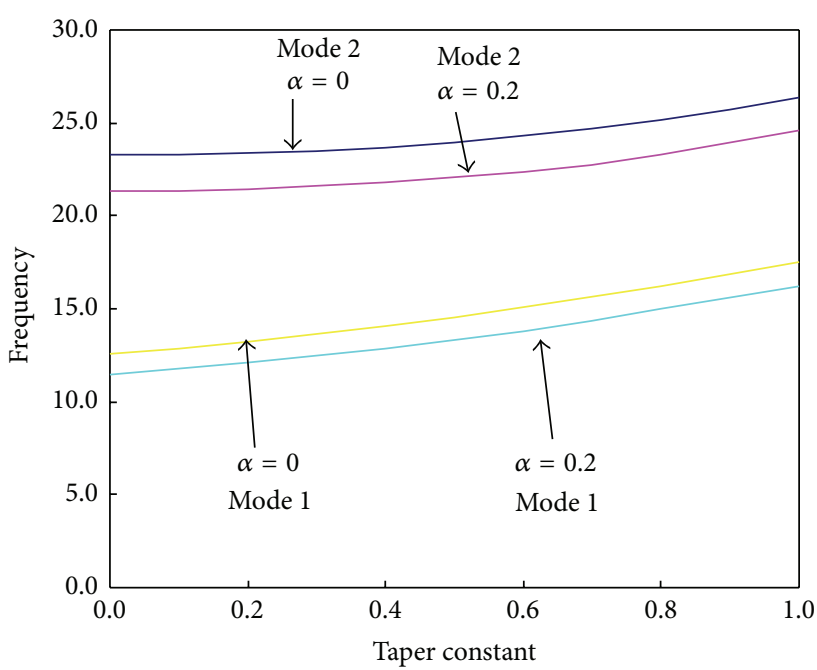

FIGURE 2: Value of frequency parameter for orthotropic trapezoidal plates for different values of taper constant and fixed values for $a / b=$ $1.0, c / b=0.75$.

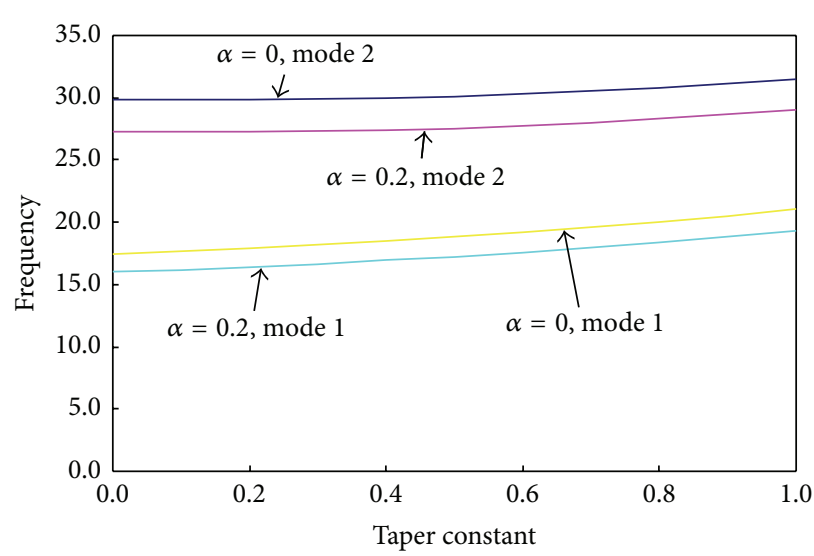

FIGURE 3: Value of frequency parameter for orthotropic trapezoidal plates for different values of taper constant and fixed values for $a / b=$ $1.0, c / b=0.5$.

are calculated for the first two modes of vibration for different values of taper constant and thermal gradient, for CSCS boundary condition.

Frequency equation (19) is quadratic in $\lambda^{2}$, so it will give two roots. The frequency is calculated for the first two modes of vibrations for the orthotropic trapezoidal plates of linearly varying thickness with thermal gradient for different values of aspect ratios.

The parameters for orthotropic material have been taken as [10]

$$
\frac{E_{2}}{E_{1}}=0.32, \quad \nu_{\xi} \frac{E_{2}}{E_{1}}=0.04, \quad \frac{G_{0}}{E_{1}}\left(1-v_{\xi} \nu_{\eta}\right)=0.09 .
$$

Verification of work is done by allowing thermal gradient and taper constant to be zero. Results are verified by comparing with the results done by Liew and Lim [11], after converting the problem in isotropic case, which confirms the 


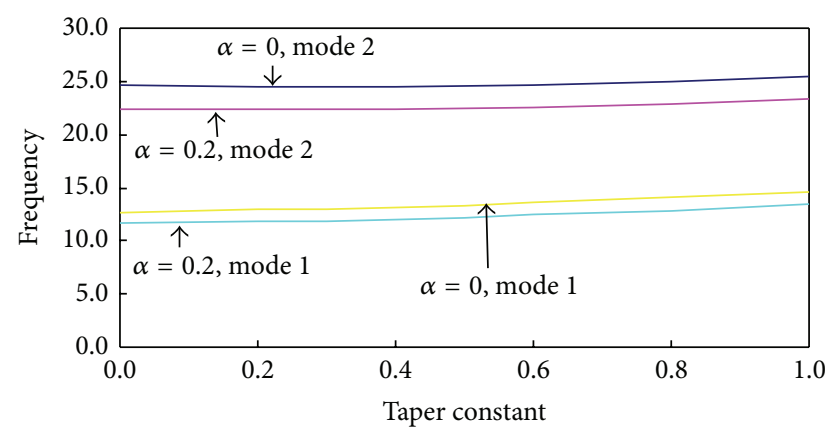

FIGURE 4: Value of frequency parameter for orthotropic trapezoidal plates for different values of taper constant and fixed values for $a / b=$ $0.75, c / b=0.5$.

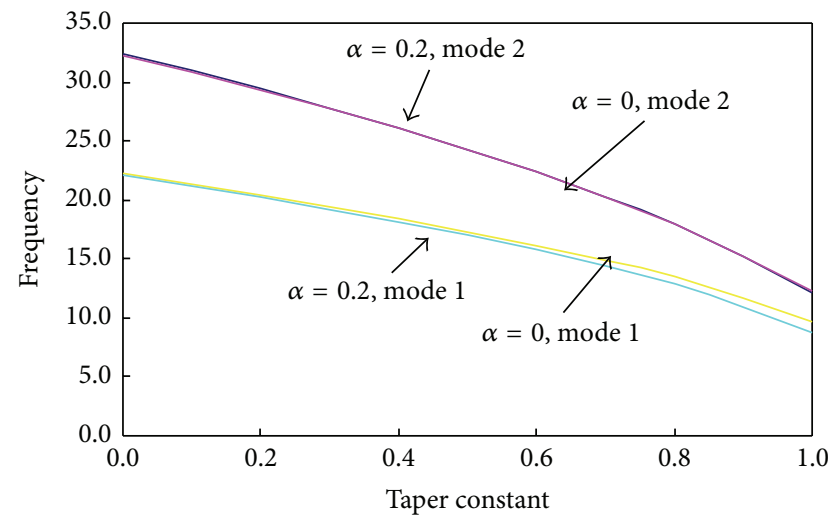

FIGURE 5: Value of frequency parameter for orthotropic trapezoidal plates for different values of taper constant and fixed values for $a / b=$ $0.75, c / b=0.25$.

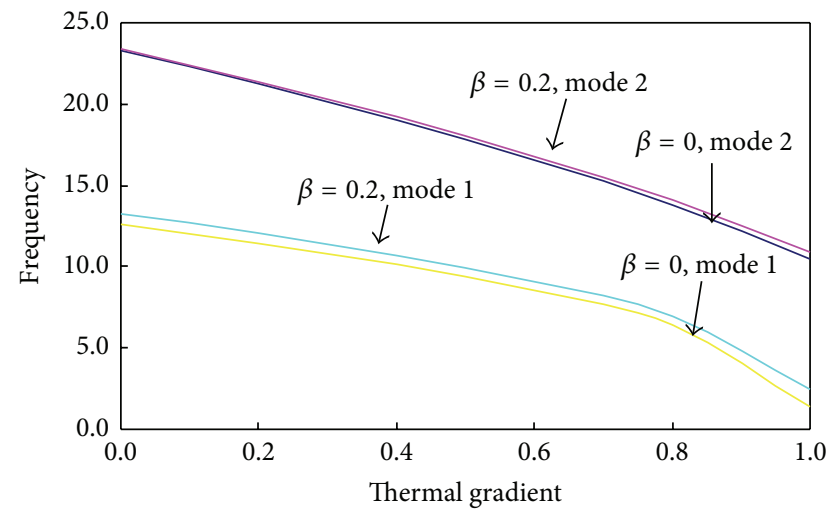

FIGURE 6: Value of frequency parameter for orthotropic trapezoidal plates for different values of thermal gradient and fixed values for $a / b=1.0, c / b=0.75$.

authenticity of work. Further, results are verified by authors that published paper [9] for taking taper constant $\beta$ to zero.

These results are plotted in Figures 2-11. A study will now be presented for a set of trapezoidal plate configurations by varying $c / b$ and $a / b$ values.

Figures 2, 3, 4, and 5 show the variation of frequency $\lambda$ with taper constant $\beta$ for various values of thermal gradient $\alpha(0.0,0.2)$ and $a / b(1 ., .75), c / b(.75, .5, .25)$. It can be concluded that, with the increase in taper constant, frequency

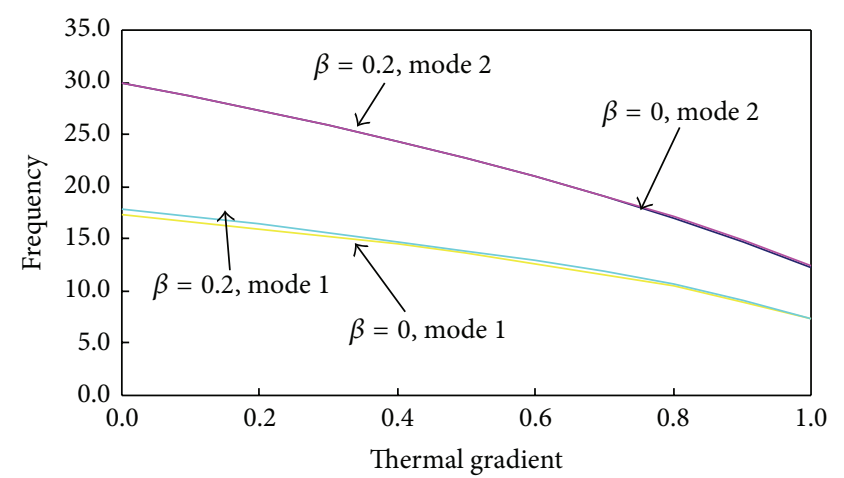

FIGURE 7: Value of frequency parameter for orthotropic trapezoidal plates for different values of thermal gradient and fixed values for $a / b=1.0, c / b=0.5$.

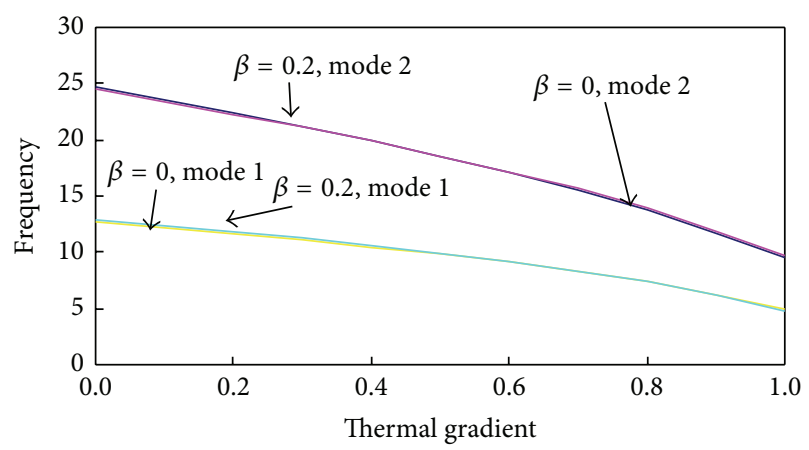

FIGURE 8: Value of frequency parameter for orthotropic trapezoidal plates for different values of thermal gradient and fixed values for $a / b=0.75, c / b=0.5$.

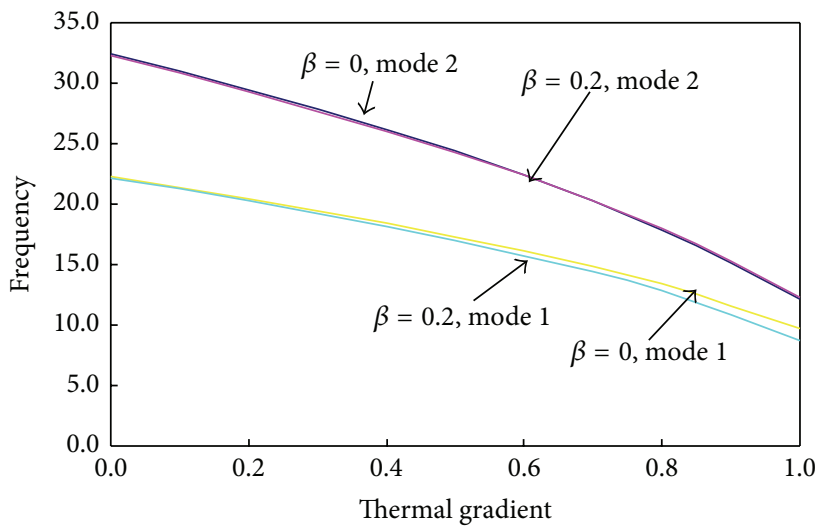

FIGURE 9: Value of frequency parameter for orthotropic trapezoidal plates for different values of thermal gradient and fixed values for $a / b=0.75, c / b=0.25$.

increases for both modes except for the case $a / b=.75$ with $c / b=.25$.

Figures $6,7,8$, and 9 show the variation of frequency $\lambda$ with thermal gradient $\alpha$ for various values of taper constant $\beta(0.0,0.2)$ and $a / b(1 ., .75), c / b(.75, .5, .25)$. It can be concluded that, with the increase in thermal gradient, frequency decreases for both modes. 


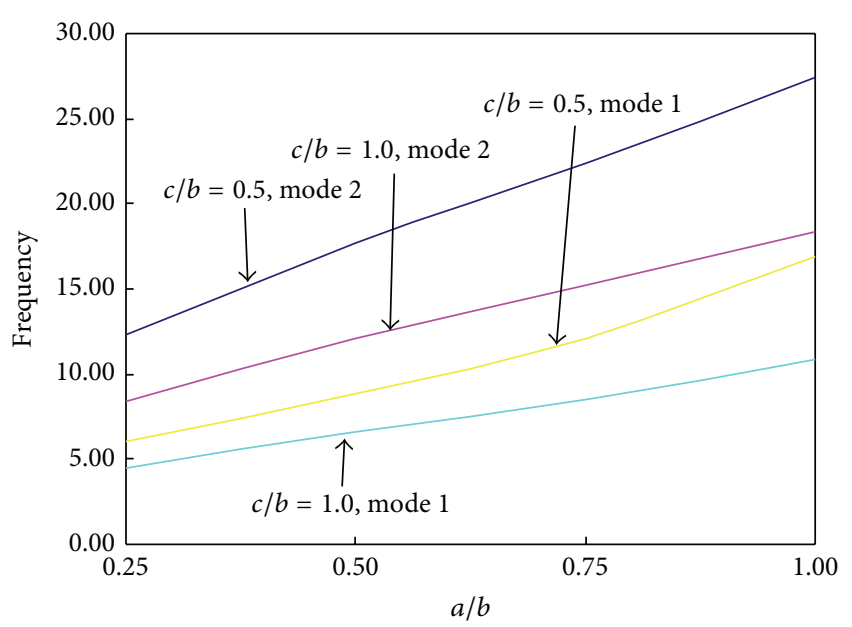

FIGURE 10: Value of frequency parameter for orthotropic trapezoidal plates for different values of $a / b$ and fixed values for $\alpha=0.2, \beta=0.4$.

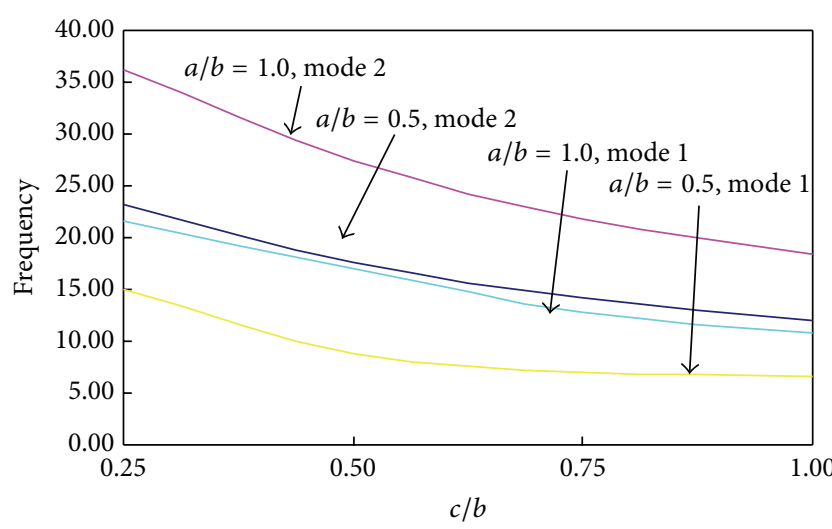

FIGURE 11: Value of frequency parameter for orthotropic trapezoidal plates for different values of $c / b$ and fixed values for $\alpha=0.2, \beta=0.4$.

Figure 10 shows the variation of frequency $\lambda$ with the aspect ratio $a / b$ for various values of $c / b$ and fixed value of $\beta, \alpha$. It can be concluded that, with the increase in the value of $a / b$, frequency increases for both modes and for all combinations of $c / b$.

Figure 11 shows the variation of frequency $\lambda$ with the aspect ratio $c / b$ for various values of $a / b$ and fixed value of $\beta, \alpha$. It can be concluded that, with the increase in the value of $a / b$, frequency decreases for both the modes and for all combinations of $c / b$.

Further, after comparing the work with authors' previous work [9], it is found that parabolically thickness variation is much better and dominated.

\section{Conflict of Interests}

The authors declare that there is no conflict of interests regarding the publication of this paper.

\section{References}

[1] A. M. Zenkour, "An exact solution for the bending of thin rectangular plates with uniform, linear, and quadratic thickness variations," International Journal of Mechanical Sciences, vol. 45, no. 2, pp. 295-315, 2003.

[2] J. S. Tomar, D. C. Gupta, and N. C. Jain, "Axisymmetric vibrations of an isotropic elastic non-homogeneous circular plate of linearly varying thickness," Journal of Sound and Vibration, vol. 85 , no. 3, pp. 365-370, 1982.

[3] J. S. Tomar, D. C. Gupta, and N. C. Jain, "Free vibrations of an isotropic non homogeneous infinite plate of parabolically varying thickness," International Journal of Pure and Applied Mathematics, vol. 15, no. 2, pp. 220-221, 1984.

[4] S. Srinivas, C. V. Joga Rao, and A. K. Rao, "An exact analysis for vibration of simply-supported homogeneous and laminated thick rectangular plates," Journal of Sound and Vibration, vol. 12, no. 2, pp. 187-199, 1970.

[5] H. T. Saliba, "Transverse free vibration of fully clamped symmetrical trapezoidal plates," Journal of Sound and Vibration, vol. 126, no. 2, pp. 237-247, 1988.

[6] R. M. Orris and M. Petyt, "A finite element study of the vibration of trapezoidal plates," Journal of Sound and Vibration, vol. 27, no. 3, pp. 325-344, 1973.

[7] M. S. Qatu, "Natural frequencies for cantilevered laminated composite right triangular and trapezoidal plates," Composites Science and Technology, vol. 51, no. 3, pp. 441-449, 1994.

[8] S. Kitipornchai, Y. Xiang, K. M. Liew, and M. K. Lim, "A global approach for vibration of thick trapezoidal plates," Computers and Structures, vol. 53, no. 1, pp. 83-92, 1994.

[9] A. K. Gupta and S. Sharma, "Thermally induced vibration of orthotropic trapezoidal plate of linearly varying thickness," JVC/Journal of Vibration and Control, vol. 17, no. 10, pp. 15911598, 2011.

[10] A. W. Leissa, Vibration of Plates, NASA SP-160, US Government Printing Office, Washington, DC, USA, 1969.

[11] K. M. Liew and M. K. Lim, "Transverse vibration of trapezoidal plates of variable thickness: symmetric trapezoids," Journal of Sound and Vibration, vol. 165, no. 1, pp. 45-67, 1993. 

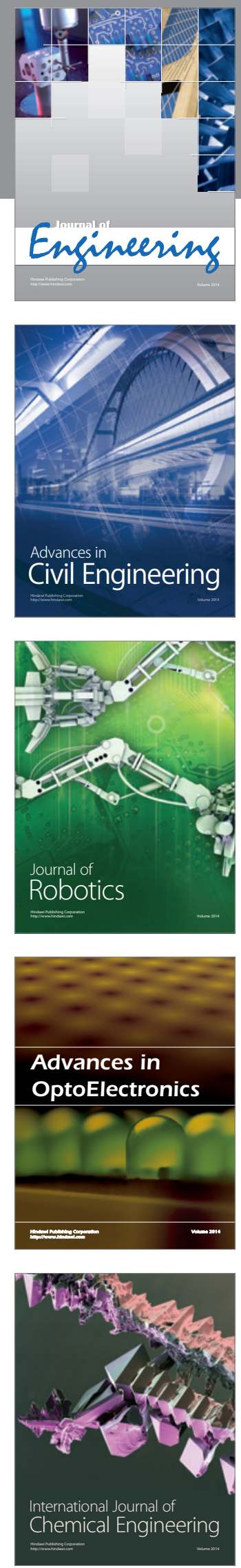

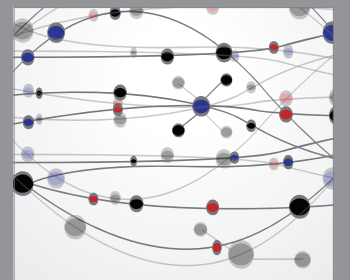

The Scientific World Journal
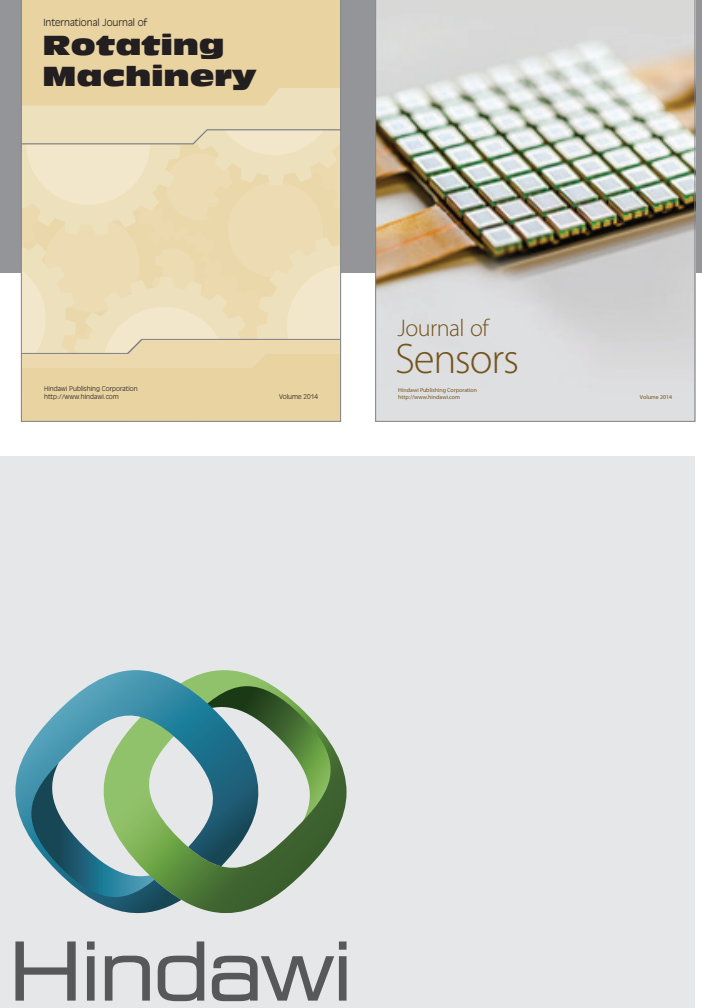

Submit your manuscripts at http://www.hindawi.com
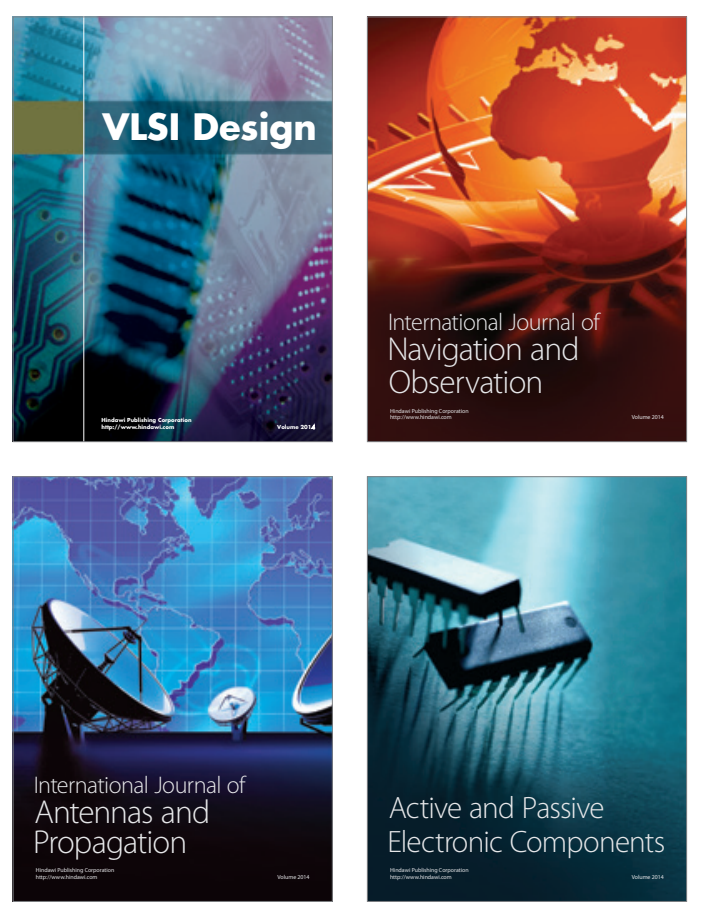
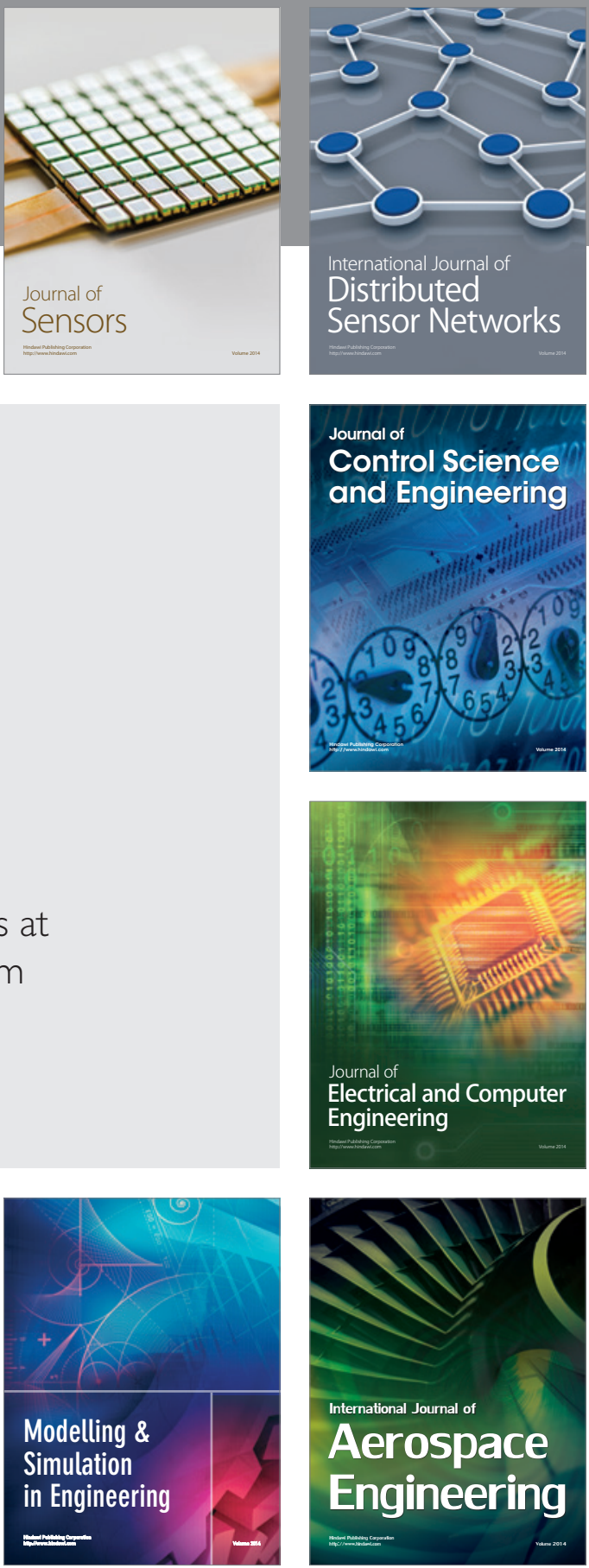

Journal of

Control Science

and Engineering
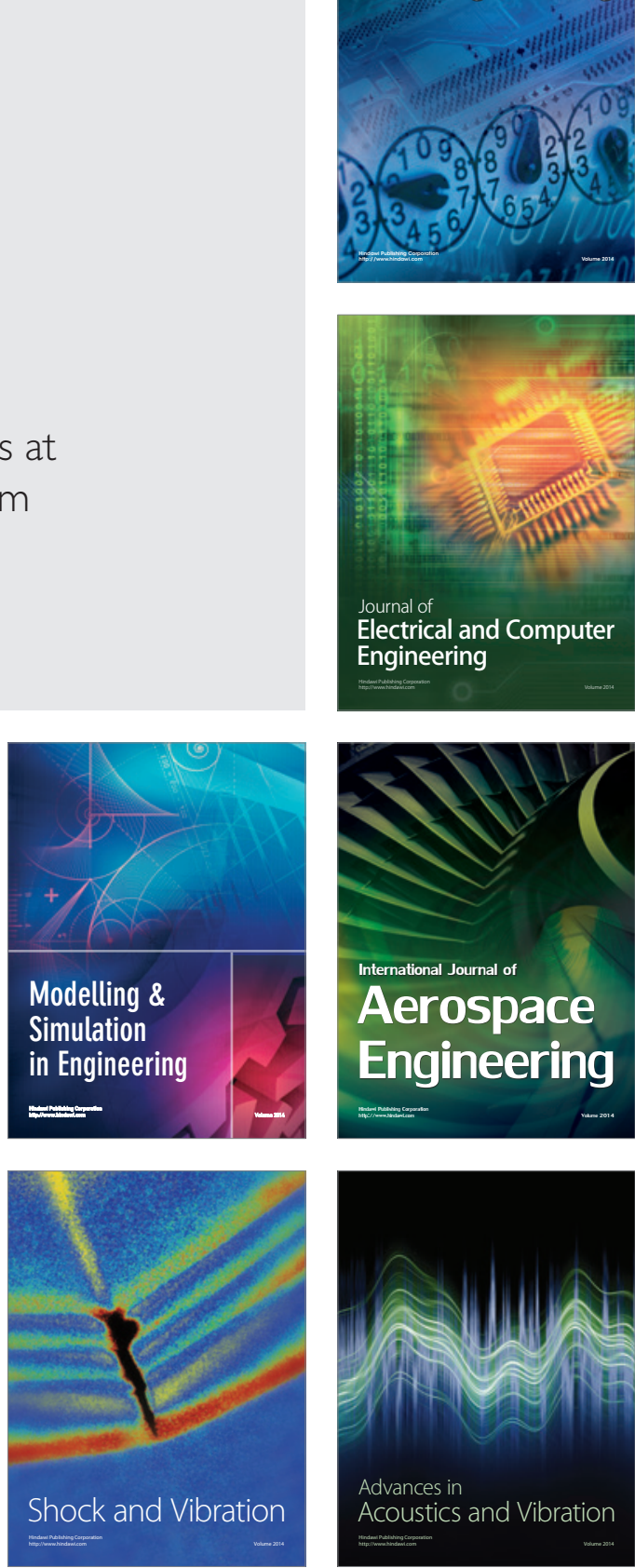